\title{
CORRECTION
}

View Article Online

View Journal | View Issue

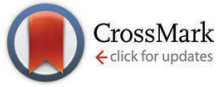

Cite this: J. Mater. Chem. C, 2016 4, 4077

DOI: $10.1039 / c 6 t c 90045 c$

www.rsc.org/MaterialsC

\section{Correction: Photochromic spiropyran- and spirooxazine-homopolymers in mesoporous thin films by surface initiated ROMP}

\author{
F. Krohm, ${ }^{a}$ J. Kind, ${ }^{\mathrm{b}}$ R. Savka, ${ }^{\mathrm{c}}$ M. Alcaraz Janßen, ${ }^{\mathrm{b}}$ D. Herold, ${ }^{\mathrm{b}}$ H. Plenio, ${ }^{\mathrm{c}}$ \\ C. M. Thiele ${ }^{b}$ and A. Andrieu-Brunsen*a
}

Correction for 'Photochromic spiropyran- and spirooxazine-homopolymers in mesoporous thin films by surface initiated ROMP' by F. Krohm et al., J. Mater. Chem. C, 2016, DOI: 10.1039/c5tc04054j.

An important reference was not cited in this article to acknowledge the work of D. J. Babu et al. ${ }^{1}$ in developing the $\mathrm{CO}_{2}$ plasma protocol that was used to treat the samples in this work. The authors of the article in J. Mater. Chem. C would also like to add D. J. Babu as a named co-worker to the acknowledgments with the revised text given below.

The Royal Society of Chemistry apologises for these errors and any consequent inconvenience to authors and readers.

\section{Acknowledgements}

We gratefully acknowledge Heike Herbert for GPC measurements and the NMR service of the Chemistry Department at the Technische Universität Darmstadt for routine NMR spectral measurements. This work was financially supported by Fonds der Chemischen Industrie, Boehringer Ingelheim Stiftung, Adolf-Messer-Stiftung, the European Research Council (ERC starting grant No. 257041) and Landes-Offensive zur Entwicklung wissenschaftlich-ökonomischer Excellenz (LOEWE) of the State of Hessen through the research initiative "Soft Control". We also acknowledge that the $\mathrm{CO}_{2}$ plasma experiments were performed by D. J. Babu in the group of Prof. J. J. Schneider at TU Darmstadt.

\section{References}

1 D. J. Babu, S. Yadav, T. Heinlein, G. Cherkashinin and J. J. Schneider, J. Phys. Chem. C, 2014, 118, 12028-12034.

\footnotetext{
${ }^{a}$ Ernst-Berl Institut für Technische und Makromolekulare Chemie, Technische Universität Darmstadt, Alarich-Weiss-Str. 4, D-64287 Darmstadt, Germany. E-mail: brunsen@cellulose.tu-darmstadt.de

${ }^{b}$ Clemens-Schöpf-Institut für Organische Chemie und Biochemie, Technische Universität Darmstadt, Alarich-Weiss-Str. 16, D-64287 Darmstadt, Germany

${ }^{c}$ Eduard-Zintl-Institut für Anorganische und Physikalische Chemie, Technische Universität Darmstadt, Alarich-Weiss-Str. 12, 64287 Darmstadt, Germany
} 